\title{
COMPARING THE GENERATION OF DTM IN A FOREST ECOSYSTEM USING TLS, ALS AND UAV-DAP, AND DIFFERENT SOFTWARE TOOLS
}

\author{
P. Crespo-Peremarch *, J. Torralba, J.P. Carbonell-Rivera, L.A. Ruiz \\ Geo-Environmental Cartography and Remote Sensing Group (CGAT), Department of Cartographic Engineering, Geodesy and \\ Photogrammetry, Universitat Politècnica de València, Camí de Vera s/n, 46022 València, Spain - pabcrepre@cgf.upv.es
}

\author{
Commission III, WG III/6
}

KEY WORDS: Digital Terrain Model, Terrestrial Laser Scanning, Airborne Laser Scanning, Unmanned Aerial Vehicle, LiDAR, LAStools, FUSION/LDV

\begin{abstract}
:
Remote sensing and photogrammetry techniques have demonstrated to be an important tool for the characterization of forest ecosystems. Nonetheless, the use of these techniques requires an accurate digital terrain model (DTM) for the height normalization procedure, which is a key step prior to any further analyses. In this manuscript, we assess the extraction of the DTM for different techniques (airborne laser scanning: ALS, terrestrial laser scanning: TLS, and digital aerial photogrammetry in unmanned aerial vehicle: UAV-DAP), processing tools with different algorithms (FUSION/LDV@ and LAStools@), algorithm parameters, and plot characteristics (canopy and shrub cover, and terrain slope). To do this, we compare the resulting DTMs with one used as reference and extracted from classic surveying measurements. Our results demonstrate, firstly, that ALS and reference DTMs are similar in the different scenarios, except for steep slopes. Secondly, TLS DTMs are slightly less accurate than those extracted for ALS, since items such as trunks and shrubs cause a great occlusion due to the proximity of the instrument, and some of the points filtered as ground correspond to these items as well, therefore a finer setting of algorithm parameters is required. Lastly, DTMs extracted for UAV-DAP in dense canopy scenarios have a low accuracy, however, accuracy may be enhanced by modifying the processing tool and algorithm parameters. An accurate DTM is essential for further forestry applications, therefore, to know how to take advantage of the available data to obtain the most accurate DTM is also fundamental.
\end{abstract}

\section{INTRODUCTION}

The importance of remote sensing and photogrammetry to characterize forest ecosystems is well known due to the many studies addressed in recent decades (Torabzadeh et al., 2014; Masek et al., 2015; Minařík and Langhammer, 2016). Among remote sensing techniques, laser scanning has played a key role in the characterization of the vertical forest structure (Lim et al., 2003; Smart et al., 2012), since laser beams go through the different vertical layers and several returns are registered by the sensor. This remote sensing technique and photogrammetry have some similarities and differences. Probably, one of the greatest common advantages over more traditional techniques is that their respective sensors may be airborne and spaceborne, collecting data over large areas. However, these techniques present some differences regarding the format of the raw data - images and laser beams for photogrammetry and laser scanning, respectively - and the methodology used to process their respective products. For instance, laser scanning beams have the potential to detect the vertical forest structure, since porosity of the vegetation allows the laser beams to go through the different strata and register them in several returns. Nonetheless, photogrammetry is more limited in detecting the vertical forest structure (Filippelli et al., 2019), since aerial images mostly capture the crown of the trees, and the rest of the vertical structure beneath them is occluded or in the shade.

Nevertheless, the potential of these techniques to characterize forest ecosystems may be reduced if a digital terrain model (DTM) with sufficient accuracy cannot be generated for the further process of height normalization (Estornell et al., 2011).
The generation of the DTM requires ground points, whose detection not only depends on the technique used, but also on the forest cover, terrain roughness and processing algorithms. For instance, proper results may be reached with low dense data whether a proper algorithm is used (Maguya et al., 2014).

Previous studies have compared the generation of DTMs by photogrammetric and laser scanning techniques. Among these studies, Gil et al. (2013) compared in a dense forest area in Canary Islands (Spain) the generation of DTMs derived from photogrammetric and airborne laser scanning (ALS) techniques using ground measures as reference. DTM derived from ALS data was especially more accurate in dense forested areas, where photogrammetric technique did not reach the ground. Wallace et al. (2016) also compared derived DTMs but generated from digital aerial photogrammetry in unmanned aerial vehicle (UAVDAP) and ALS data in a native dry sclerophyll eucalypt forest in Tasmania (Australia). Despite using photogrammetry from $\mathrm{UAV}$, they found similar results than in previous studies where terrain detection was more limited for photogrammetry especially beneath denser canopy covers. Goodbody et al. (2019) also agreed on considering the DTM derived from UAV-DAP as inaccurate in forest areas, and hence they used the DTM derived from ALS to normalize heights from UAV-DAP data. Therefore, despite the use of closer photogrammetric techniques such as UAV-DAP, which is a promising technique for the characterization of forest ecosystems, derived DTMs are inaccurate compared to those derived from ALS in denser canopy covers. However, can the difference in accuracy between ALS and UAV-DAP techniques be reduced by using different

\footnotetext{
* Corresponding author
} 
algorithms to identify ground points? Moreover, also considering terrestrial laser scanning (TLS) technique, is there a significant difference in derived DTMs compared to ALS?

The main goal of this manuscript is to compare the DTMs generated in a Mediterranean forest ecosystem with different canopy covers and terrain roughness by using ALS, TLS and UAV-DAP techniques, and algorithms implemented in two different processing tools: LAStools@ and FUSION/LDV@. To do this, the resulting DTMs derived from the different techniques and processing tools are assessed by comparing them with a reference DTM generated with classic surveying measurements.

\section{STUDY AREA AND DATA}

\subsection{Study area}

The study area is located in the Natural Park of Sierra de Espadán, which is a Mediterranean forest in the eastern Spanish province of Castellón (Figure 1). The region is highly mountainous, with presence of steep slopes only interrupted by some terraces of old crops (see Figure 2). The dominant species are maritime pine (Pinus pinaster), Aleppo pine (Pinus halepensis), and cork oak (Quercus suber). The canopy cover along the study area is variable, from $30 \%$ to $70 \%$. This percentage depends on the number of trees per unit area as well as on the density of the crowns. The most common shrub species in the study area are gorse (Genista scorpius), rosemary (Rosmarinus officinalis), grey-leaved cistus (Cistus albidus), kermes oak (Quercus coccifera), tree heath (Erica arborea), and common smilax (Smilax aspera). The presence of shrubs along the study area is also variable, from $10 \%$ to $70 \%$. This variability mainly depends on the soil properties and moisture, and on the canopy species, which hinder more or less the entrance of light (see Figure 2).

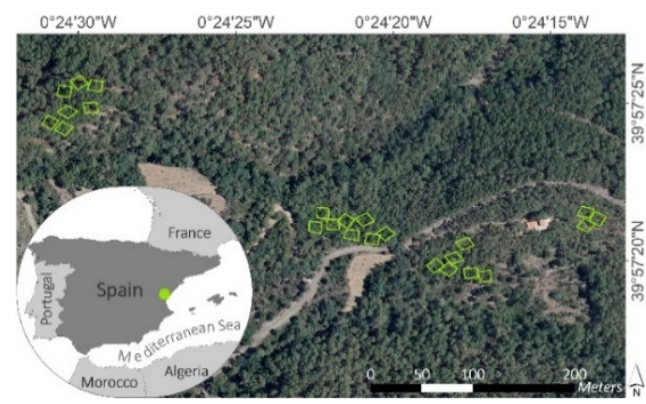

Figure 1. Location of the study area and experimental plots

\subsection{Field data}

A total of 24 square plots of $10 \mathrm{~m}$ side with a variable canopy and shrub cover and terrain slope were established in the study area (Table 1). For each plot, the degree of canopy and shrub cover was measured according to the projected area on the ground of the tree crowns and shrubs, respectively, and the position of each tree and shrub on the ground measured by GPS and classic surveying measurements in November 2019. The GPS model used during the field campaign was a Leica GNSS 1200 with an RTK accuracy of $\pm(10 \mathrm{~mm}+1 \mathrm{ppm})$ and $\pm(20 \mathrm{~mm}+1 \mathrm{ppm})$ in horizontal and vertical, respectively. Moreover, the total station for classic surveying measurements was a Topcon GTS-229 with an accuracy of $\pm(2 \mathrm{~mm}+2 \mathrm{ppm})$ and an angular accuracy of $27^{c c}$.

\subsection{Airborne laser scanning data}

The ALS data were acquired in September 2015 using a LiteMapper 6800. There is a date difference between ALS and the rest of data, however, the forest and shrub cover, and

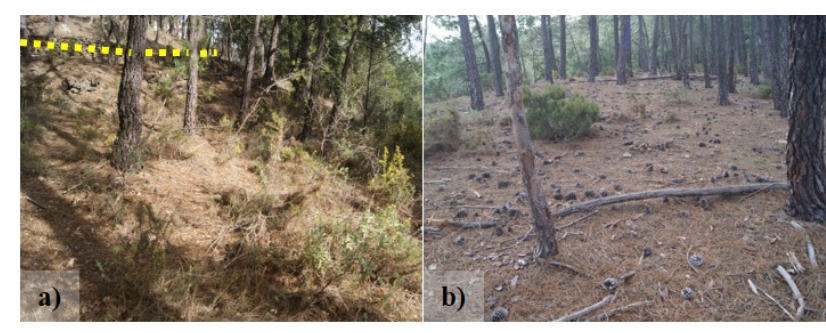

Figure 2. Examples of differences in the terrain slope and shrub cover, representing (a) a terrace of an old crop (yellow dashed

line) with presence of shrubs, and (b) a steep terrain with absence of shrubs

\begin{tabular}{|c|c|c|c|c|c|c|c|}
\hline 目 & 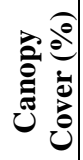 & $\frac{\partial}{b}$ & $\begin{array}{l}\frac{0}{\partial} \\
\frac{\check{a}}{\sigma}\end{array}$ & $\begin{array}{l}\text { 严 } \\
\frac{0}{a}\end{array}$ & 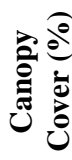 & 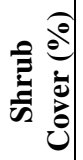 & 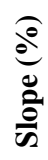 \\
\hline P25_SP001 & 50 & 30 & 30 & SP1001 & 60 & 60 & 17 \\
\hline P25_SP002 & 60 & 30 & 30 & SP1002 & 30 & 40 & 21 \\
\hline P25_SP003 & 30 & 70 & 28 & SP1003 & 30 & 30 & 9 \\
\hline P26_SP001 & 60 & 20 & 42 & SP1004 & 40 & 15 & 12 \\
\hline P26_SP002 & 50 & 30 & 38 & SP1005 & 80 & 60 & 20 \\
\hline P26_SP003 & 60 & 20 & 36 & SP1006 & 80 & 50 & 15 \\
\hline P27_SP001 & 70 & 40 & 48 & SP1007 & 70 & 40 & 28 \\
\hline P27_SP002 & 70 & 30 & 42 & SP1008 & 70 & 40 & 30 \\
\hline P27_SP003 & 80 & 50 & 46 & SP1009 & 70 & 50 & 30 \\
\hline P41_SP001 & 70 & 70 & 10 & SP1011 & 40 & 30 & 42 \\
\hline P41_SP002 & 30 & 40 & 12 & SP1012 & 60 & 10 & 24 \\
\hline P41_SP003 & 70 & 30 & 17 & SP1013 & 70 & 20 & 26 \\
\hline
\end{tabular}

Table 1. Plot characteristics

especially the terrain, which is the object of analysis in this manuscript, have not significantly changed during this period. Therefore, the use of older ALS data is valid to be compared with more current data. The study area was registered from a flight altitude between 600 and $820 \mathrm{~m}$ above ground level, an average speed of 95 knots, a pulse frequency of $300 \mathrm{kHz}$, a wavelength of $1,550 \mathrm{~nm}$, and a scan angle of $\pm 30^{\circ}$. This leaded to an average pulse density of 14 pulses $\mathrm{m}^{-2}$. Flight trajectories and the ALS point cloud were georeferenced by using the airborne GPS and inertial measurement unit (IMU), and permanent base GPS stations located near the study area. Afterwards, the accuracy in the georeferencing of the point cloud was assessed by some control points measured by GPS in flat and uncovered areas such as parking lots. The accuracy in the georeferencing of the ALS point cloud was equal to $0.043 \mathrm{~m}$.

\subsection{Terrestrial laser scanning data}

The TLS data were collected using a 3D laser scanner Trimble TX8 in November 2019. This device emits a wavelength of 1,500 $\mathrm{nm}$, a field-of-view (FOV) of $360^{\circ} \times 317^{\circ}$, an angular accuracy of $80 \mu \mathrm{rad}$, and a range systematic error of less than $2 \mathrm{~mm}$. Given that the experimental plots had a small size (i.e., $10 \mathrm{~m} \times 10 \mathrm{~m}$ ), they were registered with a single scan located in the center. This leaded to an average point density of 8,571 points $\cdot \mathrm{m}^{-2}$. Each scan identified at least three spherical targets common with adjacent scans. These spherical targets were georeferenced by classic surveying measurements at the same time the field data were collected. Afterwards, the TLS point clouds were georeferenced by identifying the different spherical targets in the point cloud, and inserting the measured coordinates in the software tool Trimble RealWorks@ v10.4. The average accuracy in the 
georeferencing of the TLS point cloud was equal to $0.031 \mathrm{~m}$.

\subsection{Digital aerial photogrammetry in unmanned aerial vehicle data}

Fieldwork was carried out in January 2020, consisting of seven flights using a DJI Phantom 3 Professional for imagery acquisition. This UAV is a quadricopter with a weight of 1,280 $\mathrm{g}$, including the built-in camera. Its maximum speed is $16 \mathrm{~m} \cdot \mathrm{s}^{-1}$, being able to fly at an altitude of up to $120 \mathrm{~m}$ from the take-off point. The camera sensor, stabilized by a three-axis gimbal, is an RGB Sony Exmor 1/2.3” CMOS of 12.4 MP, equipped with a lens FOV of $94^{\circ}, 20 \mathrm{~mm}$ focal length, $\mathrm{f} / 2.8$ focal ratio, and focus to infinite. The maximum resolution of this sensor is $4,000 \times 3,000$ pixels.

The flight plan was done on site due to the steep slope of the study area, which made it difficult to create a safe cabinet planning. Therefore, a preliminary flight was done on each study area to plan the trajectory and altitude. The flight plan was carried out using the app Pix4DCapture installed on a Samsung Galaxy Tab A. The study area was acquired with at least $80 \%$ forward and lateral overlap between images. Each flight had two different shot configurations: one with a zenithal shot and another with an oblique shot $\left(65^{\circ}\right)$. A total of seven flights with the two shot configurations were carried out to cover all the plots, and they were as follows: an area of 16.73 ha covered with 170 images, an area of 7.01 ha covered with 143 images, and an area of 29.89 ha covered with 348 images. This resulted in three different areas covered from 50 to $100 \mathrm{~m}$ height, depending on the terrain slope. Additionally, during data collection, 17 targets were located in uncovered areas along the covered area, and measured by the GPS unit described in section 2.2 as Ground Control Points (GCPs). The resulting average accuracy in the georeferencing procedure was equal to $0.059 \mathrm{~m}$.

\section{METHODS}

The different steps described in this section, and represented in Figure 3, can be divided into: (i) data georeferencing in a common coordinate system, (ii) accuracy computation of ground-truth data, (iii) computation of the derived point cloud from UAV-DAP data, (iv) extraction of ground points, $(v)$ generation of the DTMs, and (vi) comparison of the different DTMs with respect to the reference.

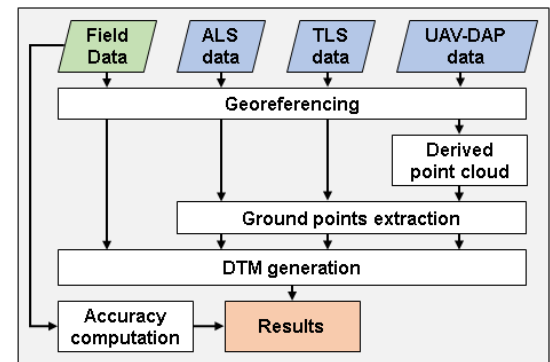

Figure 3. Overview of the methodological approach

\subsection{Computation of ground-truth data}

As described in section 2.2, we measured ground points with GPS and classic surveying measurements. It is essential that all the data sources (i.e., ALS, TLS, UAV-DAP and reference data) are in the same coordinate system to be compared. To calculate the coordinates of the ground-truth data in the corresponding coordinate system (i.e., ETRS89 UTM 30N), the process followed was (see Figure 4): (i) we measured two external points by GPS $\left(\mathrm{GPS}_{1}\right.$ and $\left.\mathrm{GPS}_{2}\right)$ in uncovered areas where a proper signal was received; (ii) we set up the total station on one of the points measured by GPS (e.g., GPS 1 ) and we observed the horizontal angle to the second point measured by GPS (i.e., $\mathrm{GPS}_{2}$ ) to calculate the offset of the horizontal angle with respect to the calculated azimuth; (iii) we measured from GPS 1 the center of one plot (e.g., SP1001) using the total station observations (see equations (1), (2), and (3)); and (iv) we measured the position of each ground point by setting up the total station on the center of the plot and measuring the distance and angles to the different points.

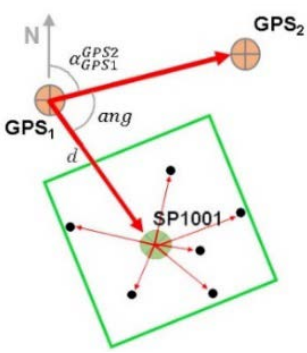

Figure 4. Depiction of the computation and georeferencing of ground-truth data; where $G P S_{1}$ and $G P S_{2}$ are two external GPS points, $\alpha_{G P S 1}^{G P S 2}$ the azimuth from $G P S_{1}$ to $G P S_{2}, d$ the distance between $G P S_{1}$ and plot center SP1001, and ang the horizontal angle from $G P S_{1}$ to $S P 1001$. Black dots represent the ground points measured (green square)

Nevertheless, despite these positions are considered as groundtruth data for this study, their errors are not null, and therefore the accuracy of the measurements must be considered before computing the differences between each DTMs and the one used as reference. Considering the process followed to compute the coordinates of ground-truth data, the two external GPS points have a known accuracy related to the GPS signal, instrument, and differential corrections. This accuracy is transmitted to the rest of the measured points by classic surveying measurements where distance, angle and set up errors of the total station were also added. Hence, this is a case of error propagation, where we calculated the accuracy related to each position point by using the approach based on Ghilani (2017) (see equations (4), (5) and (6)). As a result, the DTM generated from the ground-truth data measured by classic surveying measurements and used as reference in this study also has an accuracy.

$$
\begin{aligned}
& X_{S P 1001}=X_{G P S 1}+d \times \sin \left(\alpha_{G P S 1}^{G P S 2}+a n g\right) \\
& Y_{S P 1001}=Y_{G P S 1}+d \times \cos \left(\alpha_{G P S 1}^{G P S 2}+a n g\right) \\
& Z_{S P 1001}=Z_{G P S 1}+\frac{d}{\tan V}
\end{aligned}
$$

where $\quad X_{S P 1001}, Y_{S P 1001}, Z_{S P 1001}=$ coordinates of SP1001

$X_{G P S 1}, Y_{G P S 1}, Z_{G P S 1}=$ coordinates of GPS1

$d$ = distance

$\alpha_{G P S 1}^{G P S 2}=$ measured azimuth between GPS1 and GPS2 ang = horizontal angle between GPS2 and SP1001 from GPS1

$V=$ vertical angle

$$
\begin{aligned}
& \sigma_{X S P 1001}^{2}=\sigma_{X G P S 1}^{2}+\sigma_{d}^{2} \times \sin ^{2}\left(\alpha_{G P S 1}^{G P S 2}+a n g\right)+d^{2} \times \\
& \cos ^{2}\left(\alpha_{G P S 1}^{G P S 2}+a n g\right) \times \sigma_{\alpha_{G P S 1}^{G P S 2}}^{2}+d^{2} \times \cos ^{2}\left(\alpha_{G P S 1}^{G P S 2}+a n g\right) \times \\
& \sigma_{\text {ang }}^{2}
\end{aligned}
$$

$\sigma_{Y S P 1001}^{2}=\sigma_{Y G P S 1}^{2}+\sigma_{d}^{2} \times \cos ^{2}\left(\alpha_{G P S 1}^{G P S 2}+a n g\right)-d^{2} \times$ 


$$
\begin{aligned}
& \sin ^{2}\left(\alpha_{G P S 1}^{G P S 2}+a n g\right) \times \sigma_{\alpha_{G P S 1}^{G P S 2}}^{2}-d^{2} \times \sin ^{2}\left(\alpha_{G P S 1}^{G P S 2}+a n g\right) \times \\
& \sigma_{\text {ang }}^{2} \\
& \sigma_{Z S P 1001}^{2}=\sigma_{Z G P S 1}^{2}+\left(\frac{1}{\tan V}\right)^{2} \times \sigma_{d}^{2}+\left[\frac{-d}{\tan ^{2} V} \times(1+\right. \\
& \left.\left.\tan ^{2} V\right)\right]^{2} \times \sigma_{V}^{2} \\
& \sigma_{\alpha_{G P S 1}^{G P S 2}}=\frac{\sqrt{\sigma_{X G P S 1}^{2}+\sigma_{X G P S 2}^{2}+\sigma_{Y G P S 1}^{2}+\sigma_{Y G P S 2}^{2}}}{d_{G P S 1}^{G P S}}
\end{aligned}
$$

\subsection{Point cloud derived from UAV-DAP data}

Initial data were available in image format for UAV-DAP, while they were in point cloud format for ALS and TLS. The generation of a DTM, requires a point cloud format as input, therefore, UAV-DAP initial format had to be converted. To do this, we used Structure from Motion (SfM) algorithms, which entails two steps. Firstly, we run an automatic detection and matching of key points among multiple images. Secondly, we estimated the entire camera parameters, along with the 3D coordinates of the key points previously matched, resulting in a sparse point cloud. After this initial process, we introduced the GCPs to tune in on the adjustment and to transform the resulting point cloud to the corresponding coordinate system. The last step was to densify the sparse point cloud. This resulted in a point cloud with an average point density of 4,631 points $\cdot \mathrm{m}^{-2}$.

\subsection{Generation of digital terrain models}

Once all the data sources (i.e., ground-truth points, ALS, TLS and UAV-DAP) are in point cloud format, we filtered terrain points and used to generate the corresponding DTMs with the different processing tools and algorithm parameters.

3.3.1 Reference digital terrain model: The altitude of ground-truth points measured during the field campaign with classic surveying measurements corresponded to ground. Therefore, no ground point filter was required in this section. Next, we computed the surface interpolation using the Kriging procedure and the altitude of the points within the plot as variable. This surface interpolation resulted in a raster format, which corresponded to the reference DTM of each plot, and whose accuracy was calculated as described in section 3.1 and its pixel size was equal to $0.5 \mathrm{~m}$.

3.3.2 FUSION/LDV@): Using the point clouds from the three techniques (i.e., ALS, TLS and UAV-DAP) separately, we firstly filtered the ground points, and secondly we generated the resulting DTMs for each plot by using FUSION/LDV( v3.80 (McGaughey, 2014). This processing tool uses an algorithm adapted from Kraus and Pfeifer (1998) to filter the ground points. The algorithm consists of an iterative process that computes weights for each point according to whether they are within a threshold. These points and weights are used to compute the interpolated surface, which changes in each iteration until no significant changes are reached. Some of the parameters that define the threshold are $w$ and $g$, and final filtering of the ground points can be checked by using a height tolerance and a focal mean filter to smooth. Among the DTM generation with FUSION/LDVC, we used different algorithm parameters for the different techniques, since the denser point cloud of TLS data makes it difficult to remove the base of the trunks. For ALS and UAV-DAP data we differentiated two tests: (i) using only $g$ and $w$ parameters equal to -1 and 1 , respectively, and (ii) using the same $g$ and $w$ parameters, a height tolerance of $0.5 \mathrm{~m}$, and a focal mean filter window of $3 \times 3 \mathrm{~m}$. For the TLS we used $g$ and $w$ parameters equal to -1 and 1 , a focal mean filter window of $3 \times 3$ $\mathrm{m}$, and three height tolerances: $0.10,0.50$ and $1 \mathrm{~m}$. Finally, a raster corresponding to the DTM with a pixel size of $0.5 \mathrm{~m}$ and using the ground points filtered was generated for each plot, technique and algorithm parameters.

3.3.3 LAStools $($ : The procedure to generate the corresponding DTMs with LAStools@ v180520 (Isenburg, 2017) was similar to the one described in section 3.3.2. We firstly classified the ground points of each plot and technique separately by using different algorithm parameters. Nevertheless, LAStools (C) uses an algorithm which is a variation of Axelsson (2000). This processing tool, instead of allowing the insertion of parameter values, allows for the selection of different parameter categories depending on the scenario (e.g., whether it is either a city or a forest) and terrain roughness. The selection of the different categories varies the step size used. In this study, we tested all the possible combinations for a forest scene between the scene (wilderness and nature) and the search categories (default, fine, extra, ultra and hyper). This resulted in a point cloud with ground points classified for the different plots, techniques and algorithm parameters. Finally, we used each point cloud to compute the corresponding DTM in raster format with a pixel size of $0.5 \mathrm{~m}$.

\subsection{Digital terrain model differences}

Once we generated the DTMs for the different plots, techniques, algorithms and algorithm parameters, we compared these products with the reference DTM obtained with ground-truth data. The procedure was to calculate the corresponding DTM minus the reference one, resulting in a raster of height differences, whose root-mean-square error (RMSE) for each plot and test combination was calculated. Given that ground-truth data had also an accuracy assigned to each plot, we corrected the resulting RMSE values by subtracting the height accuracy calculated for each plot to the corresponding RMSE value, and assigning a value of zero to the negative values. Additionally, in order to assess the differences according to the canopy and shrub cover, and the terrain slope, we divided these three plot characteristics into three categories. We classified as dense all the plots with a percentage of canopy cover above or equal to $80 \%$, between $50 \%$ and $80 \%$ as moderate, and below $50 \%$ as sparse. Regarding the shrub cover, we classified those plots with a percentage above or equal to $70 \%$ as dense, between $40 \%$ and $70 \%$ as moderate, and below $40 \%$ as sparse. Finally, we classified as steep all the plots with a percentage of slope above $30 \%$, between $20 \%$ and $30 \%$ as moderate, and below $20 \%$ as slight.

\section{RESULTS}

The ground-truth points have a different accuracy, which depends on the accuracy of the external GPS points measured, and the classic surveying measurements. Table 2 shows the average height accuracy computed for each plot. The average height accuracy of all the plots is $0.132 \mathrm{~m}$ with a standard 
deviation of $0.044 \mathrm{~m}$, a minimum value of $0.088 \mathrm{~m}$ for plot P27_SP001, and a maximum value of 0.213 for plot SP1004.

\begin{tabular}{|c|c|c|c|}
\hline$\frac{\theta}{\frac{0}{2}}$ & 总冚 & 光 & 矛 \\
\hline P25_SP001 & 0.093 & SP1001 & 0.200 \\
\hline P25_SP002 & 0.091 & SP1002 & 0.203 \\
\hline P25_SP003 & 0.091 & SP1003 & 0.203 \\
\hline P26_SP001 & 0.089 & SP1004 & 0.213 \\
\hline P26_SP002 & 0.090 & SP1005 & 0.16 \\
\hline P26_SP003 & 0.090 & SP1006 & 0.142 \\
\hline P27_SP001 & 0.088 & SP1007 & 0.112 \\
\hline P27_SP002 & 0.089 & SP1008 & 0.100 \\
\hline P27_SP003 & 0.089 & SP1009 & 0.124 \\
\hline P41_SP001 & 0.170 & SP1011 & 0.116 \\
\hline P41_SP002 & 0.171 & SP1012 & 0.106 \\
\hline P41_SP003 & 0.171 & SP1013 & 0.154 \\
\hline
\end{tabular}

Table 2. Average height accuracy for the ground-truth points of each plot

The different techniques used in this study (i.e., ALS, TLS, UAV-DAP) differently register the forest vertical structure due to the location of the instrument and the measurement procedure. Two contrasted plots are represented in Figure 5, having plot P27_SP003 (Figure 5a) a dense canopy cover, moderate shrub cover, and steep terrain slope; while plot SP1003 (Figure 5b) has a sparse canopy and shrub cover, and a slight terrain slope. In a dense canopy cover, UAV-DAP is clearly blocked by the dense dominant strata, while ALS and TLS are capable of registering the crown, trunks, shrubs and terrain. This is clearly observed in the density vertical profile, where no points from UAV-DAP are registered below a height of $\sim 7 \mathrm{~m}$. On the other hand, despite the point cloud transect of plot SP1003 (Figure 5b) seems to represent a dense canopy cover due to the large number of points in the upper strata, this canopy is sparse and open, as it may be observed in the detection of the shrubs and terrain by means of UAV-DAP. In this case, UAV-DAP still registers a great number of points in the dominant strata, however, it is also capable of registering the ground as ALS and TLS.

Figure 6 shows the difference quantified by the RMSE between the reference DTM generated from the ground-truth data and those generated for the different techniques (i.e., ALS, TLS and UAV-DAP), processing tools (i.e., FUSION/LDV@ and LAStools(C), and algorithm parameters. This figure represents how differences vary according to the technique and processing tool, but also shows how different algorithm parameters modify results for a same processing tool. Larger differences with respect to the reference DTM are found for UAV-DAP. Overall, ALS differences are smaller than those of TLS, however, the minimum values for these techniques are close to zero. Regarding processing tool results, LAStools $\mathbb{C}$ generally reaches slightly smaller differences, however, overall differences for UAV-DAP show the opposite.

Figure 7 shows more specifically the differences represented in Figure 6 but grouped by categories of canopy and shrub cover, and terrain slope. Overall, the same observations than in Figure 6 are found, however, there are some interesting subtle differences. Regarding the different techniques, overall differences for UAV-DAP are reduced as canopy cover and terrain slope percentages are reduced. For the shrub cover, overall differences are not altered, however, minimum
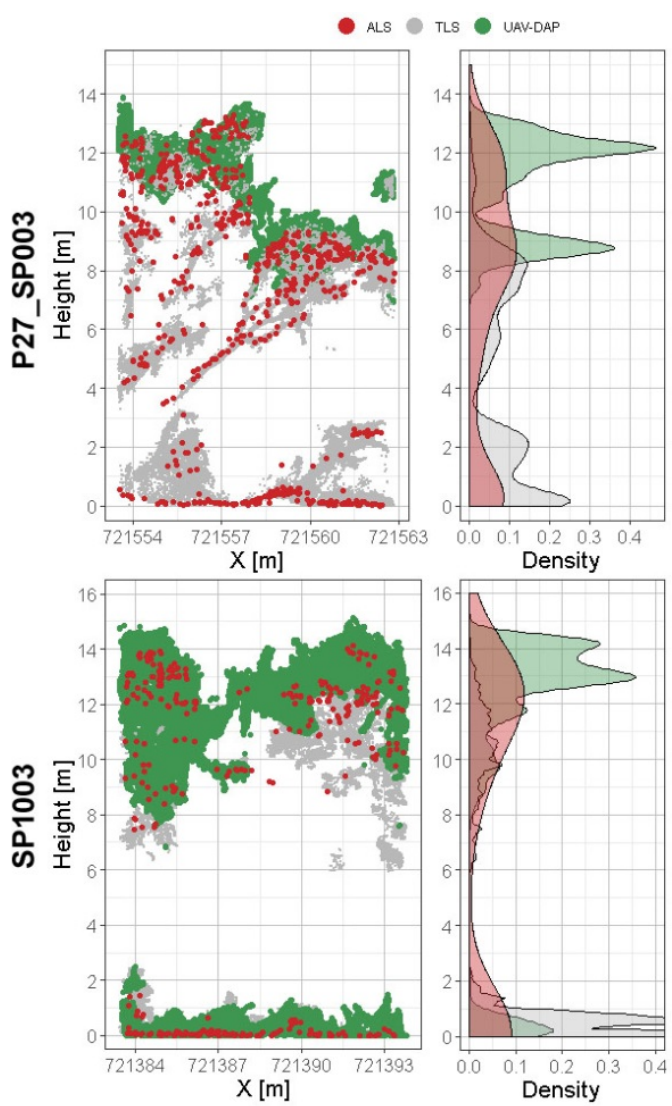

Figure 5. Vertical profiles representing from left to right: (i) a point cloud transect of $1.5 \mathrm{~m}$ wide, and (ii) the density computed from the point cloud transect according to the number of points. These profiles represent for two contrasted plots, (a) P27_SP003 and (b) SP1003

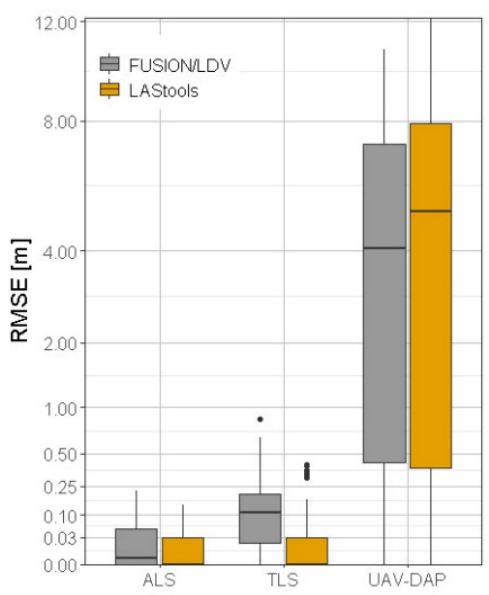

Figure 6. Box and whiskers plot representing the RMSE between the DTMs extracted using ALS, TLS and UAV-DAP, and the reference DTM, using different processing tools (i.e.,

FUSION/LDV@ and LAStools $($ ) and different algorithm parameters

differences are increased as shrub cover increases. Differences computed for ALS show that canopy cover and terrain slope have a slight influence, since the differences for the different algorithm and algorithm parameters show less dispersed results as the canopy cover and the terrain slope are sparse and slight, respectively. Nevertheless, differences become more dispersed 


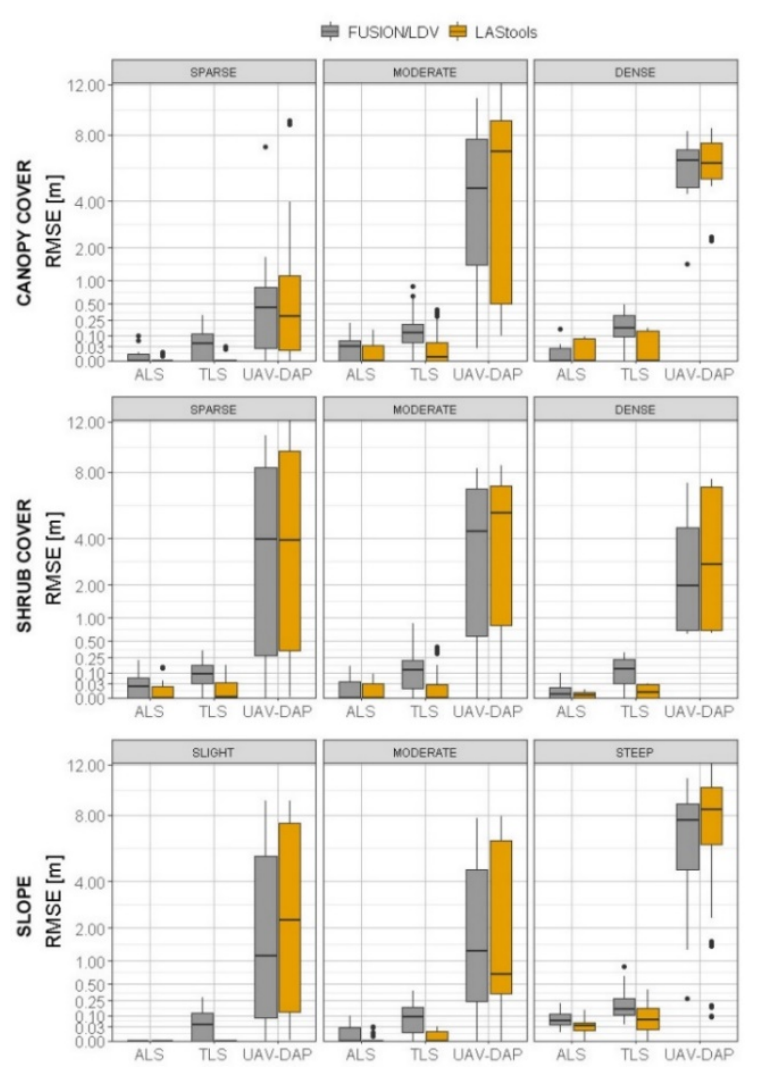

Figure 7. Box and whiskers plot representing the RMSE between the DTMs extracted from the acquisition techniques (i.e., ALS, TLS and UAV-DAP) and the reference DTM, using different processing tools (i.e., FUSION/LDV@ and

LAStools@), different algorithm parameters and grouped by different categories of canopy, shrub cover and slope

as the canopy cover and terrain slope increase, and they even increase for a steep slope. However, the presence of shrubs hardly affects the DTMs generated for ALS, resulting in less dispersed differences as the shrub cover increases. For the TLS differences, they are more dispersed as the canopy cover increases, and they increase as the shrub cover and the terrain slope increase. As showed in Figure 6, differences for LAStools $₫$ are generally smaller than those for FUSION/LDVC. However, Figure 7 also shows the opposite for the UAV-DAP technique. More specifically, this is observed for the moderate and dense canopy cover. However, the minimum differences are similar for the sparse canopy cover, the different shrub covers, and the slight and moderate slopes; and the minimum differences reached for LAStools $₫$ are smaller for steep slopes.

Figure 8 also shows RMSE differences more specifically than Figure 6, showing results from the different algorithm parameters. Again, RMSE values reached with LAStools@ are smaller than with FUSION/LDV@ for ALS and TLS, and the opposite for UAV-DAP. Regarding the different algorithm parameters, results are similar for LAStools $@$ for ALS and TLS, being nature and default slightly smaller; however, the RMSE values increase for UAV-DAP. For FUSION/LDV@, RMSE values are smaller when the mean local filter and the height tolerance are used for ALS and UAV-DAP (i.e., F2), being RMSE bigger for UAV-DAP. Finally, for TLS, the use of the smaller height tolerance (i.e., $0.10 \mathrm{~m}$ ) found the smallest RMSE.
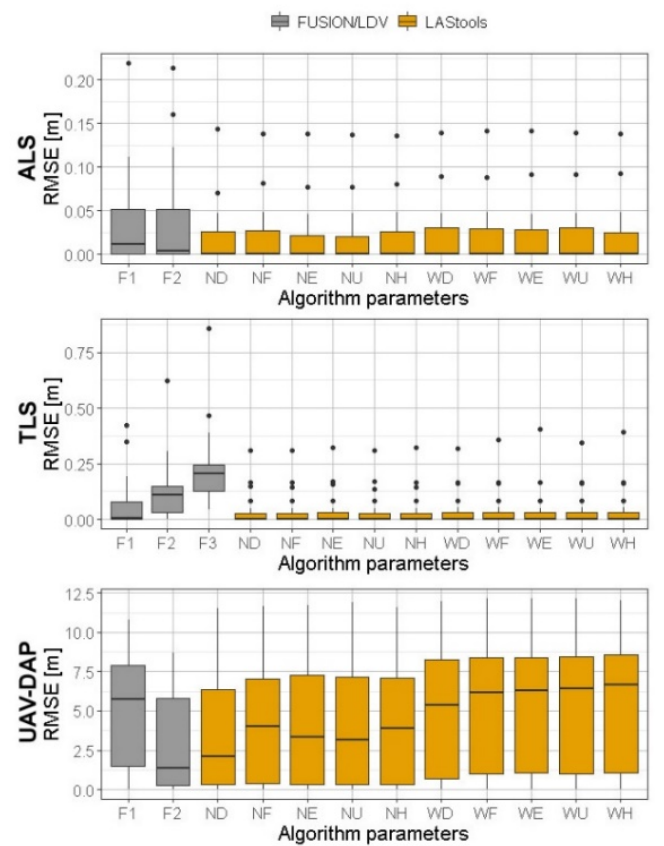

Figure 8. Box and whiskers plot representing the RMSE between the DTMs extracted from the acquisition techniques (i.e., ALS, TLS and UAV-DAP) and the reference DTM, using

different processing tools (i.e., FUSION/LDV৫ and LAStools $($ ) and different algorithm parameters. Abbreviations: F1: $g=-1$ and $w=1$ for ALS and UAV-DAP, and also the focal mean filter and a height tolerance of $0.10 \mathrm{~m}$ for TLS; F2: $g=-1$, $w=1$, a focal mean filter and a height tolerance of $0.50 \mathrm{~m}$; F3: $g$ $=-1, w=1$, a focal mean filter and a height tolerance of $1 \mathrm{~m}$; $\mathrm{N}$ : nature; W: wilderness; D: default: F: fine; E: extrafine; U: ultrafine; $\mathrm{H}$ : hyperfine

Figure 9 shows height differences between the corresponding DTM and the reference one at pixel-level. These height differences are showed for three plots with different characteristics (i.e., P25_SP003, P26_SP002 and SP1006), the different acquisition techniques, and processing tools. The height differences are not uniform along the plot, varying according the different items present (i.e., tree crowns and shrubs). Again, UAV-DAP differences are higher for intermediate and dense canopy covers (plots P26_SP002 and SP1006). However, in plot SP1006 FUSION/LDVC differences are slightly smaller. For the rest of results differences observed are not significantly different, performing slightly smaller differences for LAStools@ and ALS.

\section{DISCUSSION}

In this manuscript, we analyzed the accuracy of the DTMs generated for different techniques (i.e., ALS, TLS and UAVDAP), algorithm and algorithm parameters, in variable canopy and shrub covers, and terrain slopes locations. Key results showed that DTMs generated for ALS have the smaller differences in the different scenarios; DTMs generated for TLS required a finer setting of algorithm parameters to obtain accurate results; and those generated for UAV-DAP are highly influenced by the canopy cover, however, the use of different algorithm parameters may reduce the differences.

Results confirm the general trend accepted by the scientific community (Gil et al., 2013; Wallace et al., 2016; Goodbody et al., 2019) that DTMs generated for UAV-DAP are limited due to the high occlusion caused by the dominant strata. This limitation 


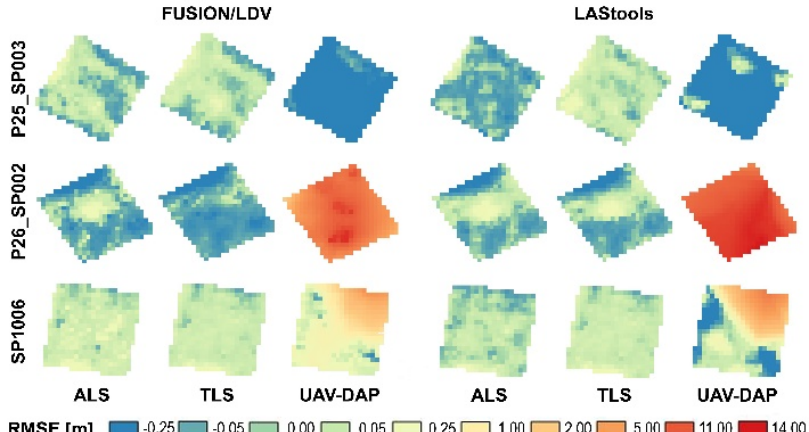

Figure 9. Height differences for three plots (P25_SP003,

P26_SP002 and SP1006) measured for the acquisition

techniques (i.e., ALS, TLS and UAV-DAP), and the processing tools (FUSION/LDV@ and LAStools@) with different algorithm parameters (for FUSION/LDV@: $g=-1, w=1$, a focal mean filter and a height tolerance of $0.50 \mathrm{~m}$ for ALS and UAV-DAP, and a height tolerance of $0.10 \mathrm{~m}$ for TLS; and for

\section{LAStools@: nature and default)}

is increased due to the SfM procedure from a passive data source such as images, where most of the pixels registered correspond to the dominant strata. Nevertheless, the UAV-DAP showed its potential to generate accurate DTMs (with a median and a minimum RMSE $\sim 0.25 \mathrm{~m}$ and equal to zero, respectively) with a dense point cloud when the canopy cover is sparse. Despite ALS is an airborne technique such as UAV-DAP, they do not present this limitation. This is due to the fact that the footprint size is small $(\sim 0.24 \mathrm{~m})$, several returns are backscattered, and it is an active sensor, so it can register beneath the dominant strata even if it is in the shade. Hence, ALS techniques may generally be used for the extraction of DTMs, while UAV-DAP techniques are recommended for sparse canopy covers.

The limitations of UAV-DAP to generate accurate DTMs in dense canopy cover scenarios results in larger errors, which are variable for the different algorithm and algorithm parameters. In the worst case scenario for the extraction of the DTM with UAVDAP technique, which is the dense canopy cover, the use of different algorithms (i.e., processing tools) and algorithm parameters shows a reduction in the differences. Smaller differences are reached by using FUSION/LDVC , since it allows greater freedom in the input of algorithm parameters. This results in removing a larger number of non-ground points, despite the density of ground points is low. Hence, the extraction of the DTM with point clouds with low density, where most of the points are blocked by the dominant strata, may be enhanced by selecting the appropriate algorithm and algorithm parameters. This coincides with Maguya et al. (2014) in pointing out that the use of a proper algorithm may reduce the influence of using a low dense point cloud.

TLS differences are surprisingly larger than those of ALS, despite the instrument registers with a denser point cloud and it is closer to the ground. Nonetheless, the proximity and high density cause some disadvantages. The proximity of the instrument to items to be registered (i.e., trunks and shrubs) increases the occlusion on the ground caused by these items. This issue is increased when single scans are used (Torralba et al., 2019). Additionally, the denser point cloud makes that more items close to the ground are registered (i.e., shrubs and trunks) (Crespo-Peremarch and Ruiz, 2017), and therefore they may wrongly be included as ground in the filtering procedure. The detection of these items with TLS involves being finer in the identification of ground points to avoid inaccurate results, as also observed by Pirotti et al. (2013). In this regard, the use of different algorithm parameters shows that TLS differences may be similar to those of ALS. Nonetheless, the detection of the ground with ALS is facilitated due to its zenithal and multi-return registration, and therefore trunks and shrubs do not cause such an occlusion as in the case of TLS. Hence, we coincide with ElAshmawy (2014) that TLS can accurately extract the DTM, however, a finer setting of the algorithm parameters than in the case of ALS is required to obtain accurate results.

The different canopy and shrub covers, and terrain slopes differently affect the different techniques. As previously mentioned, denser canopy covers affect the extraction of the DTM for UAV-DAP, however, ALS is slightly influenced, since the laser beams are capable of penetrating the dense canopy cover. More surprising is that TLS differences are also influenced by the canopy cover, since it does not interfere in the detection of the ground. This is due to the fact that plots with a denser canopy cover have a denser shrub cover, which influences on the TLS results. In addition, an increment of the canopy cover is also related to an increment of the number of trunks, which influences on the detection of the ground for TLS due to occlusion and filtering wrong points as ground. Regarding the influence of the shrub cover, it blocks the detection of the ground with UAVDAP. Again, most of the pixels from UAV-DAP images detect the shrubs, and therefore the ground is not detected. For the TLS technique, shrub cover slightly influences on the differences, since the proximity and oblique registration of the ground by means of the laser beams makes that shrubs occludes the soil. However, laser beams from ALS are almost vertical, and several returns are registered, which minimizes the influence of shrub cover on the ground detection. Finally, all the techniques are influenced by the terrain slope. The presence of more complex terrains, such as terraces with sudden changes in slope, steep terrains, or presence of mounds and holes, reduces the accuracy of DTMs. Hence, the influence of the canopy and shrub cover on the extraction of the DTM depends on the technique used, however, the influence of the terrain slope is present in all the techniques.

An accurate DTM is essential for forestry applications, since they require height normalization prior to any further analyses. Nevertheless, this is not always available, because of a lack of available data and economic issues. Firstly, it is essential to analyze the characteristics of our study area (i.e., canopy and shrub cover, and terrain slope) to estimate the derived DTM for our available data. Secondly, if the canopy and shrub covers are not dense, we can generate a DTM with an accuracy below 0.10 $\mathrm{m}$ for a more economic technique such as UAV-DAP. The key of squeezing this technique, or others with a lower ground point density, is setting the proper algorithm parameters to remove non-ground points. Other less affordable techniques, such as ALS and TLS, have fewer limitations regarding the occlusion and derived DTMs are close to the one derived for classic surveying measurements. Nevertheless, a finer setting of algorithm parameters must also be considered for TLS due to the occlusion caused on the ground by close items with a single scan in plots with dense and moderate shrubs density.

\section{CONCLUSIONS}

In this manuscript, we assessed the accuracy in the extraction of the DTM according to different techniques (i.e., ALS, TLS and UAV-DAP), algorithm and algorithm parameters, and plot characteristics. We concluded that (i) ALS technique generates DTMs with an accuracy equal to those extracted from classic surveying measurements in the different scenarios, except for a 
steep slope; (ii) the extraction of the DTM from TLS is influenced by its proximity and high point density, trunks and shrubs causing a great occlusion on the ground and a misclassification of the points corresponding to the base of tree trunks as ground, therefore more scans and/or a finer setting of the algorithm parameters is required; and (iii) despite the high influence of dense canopy covers on the extraction of the DTM for UAV-DAP, more accurate results may be reached by modifying the algorithm and algorithm parameters. The generation of an accurate DTM is essential for forestry applications. Hence, if an accurate DTM cannot be provided, due to the available data and economic issues, it is essential to know how to take advantage of the available data by setting the algorithm parameters.

\section{ACKNOWLEDGEMENTS}

The authors are thankful for the financial support provided by the Spanish Ministerio de Economía y Competitividad and FEDER, in the framework of the project CGL2016-80705-R.

\section{REFERENCES}

Axelsson, P., 2000. DEM generation from laser scanner data using adaptive TIN models. International Archives of Photogrammetry and Remote Sensing, 33(4), 110-117. doi.org/10.1080/17538947.2010.533201

Crespo-Peremarch, P., Ruiz, L.A., 2017. Análisis comparativo del potencial del ALS y TLS en la caracterización estructural de la masa forestal basado en voxelización. In XVII Congreso de la Asociación Española de Teledetección: Nuevas Plataformas y Sensores de Teledetección, 131-135.

El-Ashmawy, K.L., 2014. A comparison between analytical aerial photogrammetry, laser scanning, total station and global positioning system surveys for generation of digital terrain model. Geocarto International, 30(2), 154-162. doi.org/10.1080/10106049.2014.883438

Estornell, J., Ruiz, L.A., Velázquez-Martí, B., Hermosilla, T., 2011. Analysis of the factors affecting LiDAR DTM accuracy in a steep urban area. International Journal of Digital Earth, 4(6), 521-538. doi.org/10.1080/17538947.2010.533201

Filippelli, S.K., Lefsky, M.A., Rocca, M.E., 2019. Comparison and integration of lidar and photogrammetric point clouds for mapping pre-fire forest structure. Remote Sensing of Environment, 224, 151-166. doi.org/10.1016/j.rse.2019.01.029

Ghilani, C.D., 2017. Adjustment computations: spatial data analysis. John Wiley \& Sons. doi.org/10.1002/9781119390664

Gil, A.L., Núñez-Casillas, L., Isenburg, M., Benito, A.A., Bello, J.J.R., Arbelo, M., 2013. A comparison between LiDAR and photogrammetry digital terrain models in a forest area on Tenerife Island. Canadian Journal of Remote Sensing, 36(5), 396-409. doi.org/10.1007/s40725-019-00087-2

Goodbody, T.R., Coops, N.C., White, J.C., 2019. Digital aerial photogrammetry for updating area-based forest inventories: A review of opportunities, challenges, and future directions. Current Forestry Reports, 5(2), 55-75. doi.org/10.1007/s40725019-00087-2

Isenburg, M., 2017. LAStools. Rapidlasso GmbH: Gilching, Germany.
Kraus, K., Pfeifer, N., 1998. Determination of terrain models in wooded areas with airborne laser scanner data. ISPRS Journal of Photogrammetry and Remote Sensing, 53(4), 193-203. doi.org/10.1016/S0924-2716(98)00009-4

Lim, K., Treitz, P., Wulder, M., St-Onge, B., Flood, M., 2003. LiDAR remote sensing of forest structure. Progress in physical geography, 27(1), 88-106. doi.org/10.1191/0309133303pp360ra

Maguya, A.S., Junttila, V., Kauranne, T., 2014. Algorithm for extracting digital terrain models under forest canopy from airborne LiDAR data. Remote Sensing, 6(7), 6524-6548. doi.org/10.3390/rs6076524

Masek, J.G., Hayes, D.J., Hughes, M.J., Healey, S.P., Turner, D.P., 2015. The role of remote sensing in process-scaling studies of managed forest ecosystems. Forest Ecology and Management, 355, 109-123. doi.org/10.1016/j.foreco.2015.05.032

McGaughey, R.J., 2014. FUSION/LDV: Software for LiDAR data analysis and visualization. Department of Agriculture, Forest Service, Pacific Northwest Research Station: Seattle, WA, USA.

Minařík, R., Langhammer, J., 2016. Use of multispectral UAV photogrammetry for detection and tracking of forest disturbance dynamics. International Archives of the Photogrammetry, Remote Sensing \& Spatial Information Sciences, XLI-B8, 711718. doi.org/10.5194/isprs-archives-XLI-B8-711-2016

Pirotti, F., Guarnieri, A., Vettore, A., 2013. Vegetation filtering of waveform terrestrial laser scanner data for DTM production. Applied Geomatics, 5(4), 311-322. doi.org/10.1007/s12518-0130119-3

Smart, L.S., Swenson, J.J., Christensen, N.L., Sexton, J.O., 2012. Three-dimensional characterization of pine forest type and redcockaded woodpecker habitat by small-footprint, discrete-return lidar. Forest Ecology and Management, 281, 100-110. doi.org/10.1016/j.foreco.2012.06.020

Torabzadeh, H., Morsdorf, F., Schaepman, M.E., 2014. Fusion of imaging spectroscopy and airborne laser scanning data for characterization of forest ecosystems - A review. ISPRS Journal of Photogrammetry and Remote Sensing, 97, 25-35. doi.org/10.1016/j.isprsjprs.2014.08.001

Torralba, J., Ruiz, L.A., Carbonell-Rivera, J.P., CrespoPeremarch, P., 2019. Análisis de posiciones y densidades TLS (Terrestrial Laser Scanning) para optimizar la estimación de parámetros forestales. In XVIII Congreso de la Asociación Española de Teledetección: Teledetección: Hacia una Visión Global del Cambio Climático, 443-446.

Wallace, L., Lucieer, A., Malenovský, Z., Turner, D., Vopěnka, P., 2016. Assessment of forest structure using two UAV techniques: A comparison of airborne laser scanning and structure from motion (SfM) point clouds. Forests, 7(3), 62. doi.org/10.3390/f7030062 